\title{
Calculated polarizabilities of small Si clusters
}

\author{
Ke Deng, ${ }^{1}$ Jinlong Yang, ${ }^{1,2}$ and C. T. Chan ${ }^{2}$ \\ ${ }^{1}$ Open Laboratory of Bond Selective Chemistry, University of Science and Technology of China, \\ Hefei 230026, People's Republic of China \\ ${ }^{2}$ Physics Department, Hong Kong University of Science and Technology, Clear Water Bay, Hong Kong \\ (Received 19 May 1999; revised manuscript received 6 October 1999; published 13 January 2000)
}

\begin{abstract}
The polarizabilities of Si clusters with 9 to 28 atoms are calculated using a density functional cluster method. The atomic geometries are based on those carefully optimized by energy minimization. The polarizability shows fairly irregular variation with cluster size, but all calculated values are higher than the polarizability of a dielectric sphere with bulk dielectric constant and equivalent volume.
\end{abstract}

PACS number(s): 36.40.-c, 42.65.An, 33.15.Kr, 33.10.Dk

The structure and physical properties of small clusters, especially that of $\mathrm{Si}$, have attracted a lot of attention in the past decade [1-12]. The physical property of a cluster is ultimately determined by its geometrical structure, and so the geometrical arrangement of the atoms is the key to the understanding of the physics of these systems. However, a direct experimental determination of the geometric structure of small cluster is very difficult, if not impossible. On the theoretical side, it is possible in principle to determine the ground state structure by a direct minimization of the total energy of the cluster with respect to the atomic coordinates. Once the ground state structure is fixed, all other physical properties can in principle be calculated. In the past two decades or so, $a b$ initio density functional based techniques and $a b$ initio quantum chemistry method have advanced to such a level that the relative energies of structural isomers can be determined to a high precision. The zero-force geometry (local minimum) of an atomic cluster can be determined by total energy and force calculations given a certain set of initial atomic coordinates. Indeed, for very small clusters, the structure can be determined theoretically. As the cluster size increases, the number of local minima increases rapidly with the number of atoms in the cluster, and it becomes impractical to exhaust all the possible local minima. Even though efficient optimization algorithms have been devised in order to overcome this problem, and atomic clusters of progressively lower energies have been proposed, there is no assurance that a particular zero-force geometry found by theory is the global ground state. Even the most efficient optimization algorithm can only cover a small part of the phase space. It is thus important to compare the physical properties of these clusters with experimentally measured values. The agreement between theoretical calculations and experimental measurements can establish the ground state geometry of small clusters.

The purpose of this article is to calculate the polarizabilities of small $\mathrm{Si}_{n}(9 \leqslant n \leqslant 28)$ clusters whose structures have been determined previously via accurate $a b$ initio total energy techniques combined with efficient optimization algorithm. The polarizabilities of small $\mathrm{Si}$ clusters have been measured by Schäfer and co-workers [6] for clusters with $n$ $=4-120$. For $n=60-120$, the measured polarizability $\alpha$ per atom is systematically less than the bulk limit, which has a value of $\alpha=3.7 \AA$ /atom. This bulk limit of $\alpha$ is defined as

$$
\alpha=\frac{3}{4 \pi} \frac{\epsilon-1}{\epsilon+2} \Omega,
$$

where $\Omega$ is the bulk atomic volume and $\epsilon$ is the bulk static dielectric constant of Si. This smaller polarizability is interpreted as the consequence of the quantum size effect of $\epsilon$ : a small ensemble of atoms should have a larger gap and hence a smaller $\epsilon$ than the bulk. However, for even smaller clusters $(9 \leqslant n \leqslant 50)$, the measured polarizabilities vary strongly and irregularly with $n$. Measured $\alpha$ values can be bigger or smaller than the bulk limit, although most of the measured $\alpha$ tend to be smaller than the bulk limit. Vasiliev, Ögüt, and Chelikowsky [7] have calculated $\alpha$ for $\mathrm{Si}_{n}$ using a pseudopotential based finite-difference method for $n \leqslant 10$ based on the structures determined by energy minimization. The calculated average $\alpha$ are all higher than the bulk polarizability, although the values tend to decrease towards the bulk limit as $n$ increases. Since almost all the experimental results are beyond this range, there is no meaningful comparison that can be made. Sieck et al. [8] and Jackson et al. [9] have calculated $\alpha$ for selected cluster sizes $(n=3-14,20,21)$, based on geometries that they optimized by calculation. Again, the calculated values are above the bulk limit, and there are large differences between the calculated and measured values. In this work, we calculate $\alpha$ from $n=9$ to 28 .

The calculations are based on local density functional formalism as implemented in a cluster simulation package (DMOL [13]). The Vosko-Wilk-Nusair parametrization of the local exchange-correlation energy is used. The all electron wave functions are expanded in a local atomic orbital basis. In the double-numerical basis set, the $\mathrm{Si} 3 s$ and $3 p$ orbitals are represented by two basis functions each, and a $3 d$ type wave function has been used to describe polarization. The extra-fine grid mesh points are used for the matrix integration. The starting geometry of the clusters with $n$ from 12 to 28 are taken from the atomic configurations that have been optimized by a genetic algorithm $[10,14]$. These cluster geometries are probably the best (i.e., lowest energy) in the literature, and there is good evidence that these structures are at least consistent with some measured quantities such as ionic mobilities [10], dissociation energies [11], and ionization potentials [12]. For smaller clusters (from $n=9$ to 13), cluster geometries provided by Sieck and co-workers are also 
used as the starting point. Symmetry constrained geometry optimizations are performed with the Broyden-FletcherGoldfard-Shanno technique from these starting geometries. This assures that the calculated dipole moments and polarizabilities would pertain to the energy minimum structure within the current formulation. In each case, the starting geometries are already very close to the final zero-force configuration within the present formalism. Once the geometries are optimized, the polarizability can be computed by monitoring the change of the dipole moment as we impose an external electric field. The atomic geometries are not further relaxed as the perturbing $E$ field is small.

The diagonal elements of the polarizability tensor $\alpha_{i i}$, defined as

$$
\alpha_{i i}=\frac{\partial \mu_{i}}{\partial E_{i}},
$$

where $\mu_{i}$ and $E_{i}$ are the Cartesian components of the dipole moment and the applied $E$ field, respectively. In our calculation, this first order change is estimated by finite difference. The dipole moment is first calculated at zero external field. An external field of strength 0.001 a.u. is then imposed in the positive and negative $x, y, z$ directions, respectively, and the change in the dipole moment is calculated and averaged to give $\alpha_{i i}$. (There are a few cases in which the $|\Delta \mu|$ for the positive and negative fields differs by more than $0.01 \mathrm{D}$. In those cases, we decrease the external field strength to 0.0005 a.u.) The average polarizability is then given by the invariant trace $\langle\alpha\rangle=\frac{1}{3}\left(\alpha_{x x}+\alpha_{y y}+\alpha_{z z}\right)$.

As a benchmark test of the calculation procedures, we computed the polarizability of the $\mathrm{CO}$ molecule. Our calculated value of $1.96 \AA^{3}$ agrees well the experimental value of $1.95 \AA^{3}$. This shows that if the geometric structure is known, the local density formalism can provide a reasonably accurate description of the linear response of small molecules under external electric fields.

Our calculated results for $\mathrm{Si}$ clusters are summarized in Table I, in which we list the binding energies, dipole moments and the average polarizabilities for Si clusters with various sizes. We have checked the LDA calculated $\langle\alpha\rangle$ values with the generalized gradient approximation (GGA) [15] for clusters with $n=9,10,20$, and found that the changes are very small (see Table I). In many cases, isomers that are competitive in energy are also considered so that we have multiple entries in Table I for some values of $n$. The ground state configuration is by definition the isomer with the lowest energy. The polarizabilities as a function of cluster size are plotted in Fig. 1. The $\langle\alpha\rangle$ values corresponding to the ground state structures are shown as solid circles, while the open circles corresponds to other isomers. The results of Vasiliev et al. and the bulk limit are also plotted on the same graph for comparison. From these results with $n$ smaller than 10, the $\langle\alpha\rangle$ values start from a large value and then apparently decreases towards the bulk limit from above. However, we see from Fig. 1 that the polarizability actually attains a minimum when $n$ is between 10 and 15. For larger cluster sizes, the polarizabilities of the ground state structures display large variations with cluster size, but the general trend has a
TABLE I. The calculated binding energies (BE), absolute values of the dipole moment $(\mu)$, average polarizabilities $(\langle\alpha\rangle)$, effective polarizabilities $\left(\alpha_{\text {eff }}\right)$ for $\mathrm{Si}_{n}$. For some clusters $\left(\mathrm{Si}_{9}, \mathrm{Si}_{10}, \mathrm{Si}_{20}\right)$, we include GGA calculated values of $\langle\alpha\rangle$ in the brackets after the corresponding LDA values. The starting geometries of the clusters marked with an astrisk are from Ref. [8], the others from Refs. $[10,14]$.

\begin{tabular}{|c|c|c|c|c|c|}
\hline$n$ & Symmetry & $\mathrm{BE}(\mathrm{eV})$ & $\mu(\mathrm{D})$ & $\begin{array}{c}\langle\alpha\rangle \\
\left(\AA^{3} / \text { atom }\right)\end{array}$ & $\begin{array}{c}\alpha_{\text {eff }} \\
\left(\AA^{3 / \text { atom })}\right)\end{array}$ \\
\hline $9 *$ & $\mathrm{C}_{2 \mathrm{v}}$ & -37.762 & 0.20 & $4.42(4.34)$ & 4.46 \\
\hline $10^{*}$ & $\mathrm{C}_{3 \mathrm{v}}$ & -43.232 & 0.72 & $4.24(4.27)$ & 4.65 \\
\hline $10^{*}$ & $\mathrm{~T}_{d}$ & -42.546 & 0.00 & 4.70 & 4.70 \\
\hline $11 *$ & $\mathrm{C}_{s}$ & -46.645 & 0.73 & 4.18 & 4.57 \\
\hline $11 *$ & $\mathrm{C}_{1}$ & -46.895 & 0.88 & 4.12 & 4.69 \\
\hline $11^{*}$ & $\mathrm{C}_{s}$ & -46.500 & 1.39 & 4.04 & 5.47 \\
\hline 12 & $\mathrm{C}_{2 \mathrm{v}}$ & -51.565 & 0.19 & 4.15 & 4.18 \\
\hline 12 & $\mathrm{C}_{s}$ & -50.934 & 0.77 & 4.53 & 4.94 \\
\hline 13 & $\mathrm{C}_{s}$ & -55.860 & 1.22 & 4.39 & 5.31 \\
\hline 13 & $\mathrm{C}_{3 \mathrm{v}}$ & -55.628 & 0.12 & 4.50 & 4.51 \\
\hline 13 & $\mathrm{C}_{2 \mathrm{v}}$ & -55.838 & 1.31 & 4.43 & 5.51 \\
\hline $13^{*}$ & $\mathrm{C}_{2 \mathrm{v}}$ & -55.381 & 0.28 & 4.17 & 4.21 \\
\hline 14 & $\mathrm{C}_{s}$ & -60.782 & 1.10 & 3.97 & 4.67 \\
\hline 15 & $\mathrm{C}_{3 \mathrm{v}}$ & -65.581 & 2.60 & 4.36 & 8.02 \\
\hline 15 & $\mathrm{D}_{3 h}$ & -65.575 & 0.00 & 4.13 & 4.13 \\
\hline 16 & $\mathrm{C}_{2 h}$ & -69.305 & 0.00 & 4.48 & 4.48 \\
\hline 16 & $\mathrm{C}_{2 h}$ & -69.328 & 0.00 & 4.67 & 4.67 \\
\hline 17 & $\mathrm{C}_{3 \mathrm{v}}$ & -74.410 & 0.80 & 4.66 & 4.96 \\
\hline 18 & $\mathrm{C}_{3 \mathrm{v}}$ & -79.113 & 4.16 & 4.86 & 12.63 \\
\hline 18 & $\mathrm{D}_{4 d}$ & -77.341 & 0.00 & 4.44 & 4.44 \\
\hline 18 & $\mathrm{C}_{1}$ & -78.894 & 0.01 & 4.66 & 4.66 \\
\hline 19 & $\mathrm{C}_{2 \mathrm{v}}$ & -83.713 & 1.01 & 4.61 & 5.05 \\
\hline 20 & $\mathrm{C}_{s}$ & -88.201 & 1.06 & $4.46(4.42)$ & 4.91 \\
\hline 20 & $\mathrm{D}_{3 h}$ & -87.815 & 0.00 & 5.13 & 5.13 \\
\hline 21 & $\mathrm{C}_{2 \mathrm{v}}$ & -91.612 & 0.60 & 4.83 & 4.97 \\
\hline 22 & $\mathrm{C}_{2 \mathrm{v}}$ & -95.716 & 0.28 & 5.08 & 5.11 \\
\hline 22 & $\mathrm{C}_{2 \mathrm{v}}$ & -97.058 & 1.50 & 4.86 & 5.69 \\
\hline 23 & $\mathrm{C}_{2 \mathrm{v}}$ & -100.879 & 0.85 & 4.81 & 5.06 \\
\hline 23 & $\mathrm{C}_{2 \mathrm{v}}$ & -100.890 & 0.13 & 5.04 & 5.05 \\
\hline 24 & $\mathrm{C}_{3 \mathrm{v}}$ & -104.759 & 4.13 & 5.08 & 10.83 \\
\hline 24 & $\mathrm{C}_{s}$ & -105.393 & 3.81 & 4.95 & 9.86 \\
\hline 24 & $\mathrm{C}_{2 \mathrm{v}}$ & -105.299 & 0.84 & 4.72 & 4.96 \\
\hline 25 & $\mathrm{C}_{3 \mathrm{v}}$ & -110.323 & 4.42 & 5.42 & 11.75 \\
\hline 25 & $\mathrm{C}_{3 \mathrm{v}}$ & -110.300 & 1.23 & 5.16 & 5.66 \\
\hline 25 & $\mathrm{C}_{3 \mathrm{v}}$ & -109.868 & 1.21 & 5.13 & 5.60 \\
\hline 26 & $\mathrm{C}_{s}$ & -114.341 & 5.70 & 5.39 & 15.52 \\
\hline 26 & $\mathrm{C}_{2 \mathrm{v}}$ & -113.713 & 0.75 & 4.63 & 4.81 \\
\hline 27 & $\mathrm{C}_{2 \mathrm{v}}$ & -118.548 & 0.50 & 5.51 & 5.58 \\
\hline 28 & $\mathrm{C}_{2 \mathrm{v}}$ & -122.877 & 1.12 & 5.57 & 5.94 \\
\hline
\end{tabular}

positive slope. The calculated values actually deviate more from the bulk limit as we go from $n=10$ to 28 . If the structure of these clusters are correct, our results show that $n$ $=28$ is still far away from the bulk limit as far as the polarizability is concerned.

We note that the experimental values also show large variations with cluster size. In this aspect, theory and experi- 


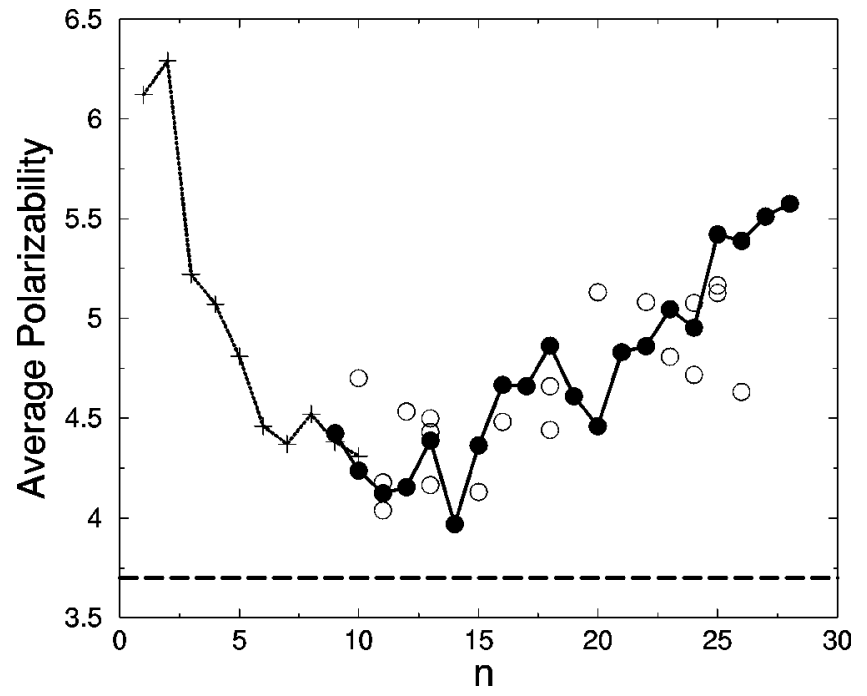

FIG. 1. The calculated average polarizability (in $\AA^{3}$ per atom) plotted against $n$, the number of atoms in the Si cluster. Solid circles connected by the lines correspond to the ground state geometries, while open circles correspond to other isomers. For completeness, we include the results of Vasiliev et al. [7] (plotted as crosses). The bulk limit is indicated by the dotted line.

ment are consistent. However, all the calculated $\langle\alpha\rangle$ values for the ground state structures are bigger than the bulk limit, while the experimental values fluctuates about bulk value. In fact, most measured values are less than the bulk limit in this regime, and some can be substantially smaller than bulk.

Since most of the clusters have a permanent dipole moment, the measured polarizability should be given (in the high temperature and low field limit) as $\alpha_{\text {eff }}=\langle\alpha\rangle$ $+(1 / 3 k T) \mu^{2}$. This means that the measured effective polarizability should be bigger than the average $\alpha$. For completeness, the effective polarizability per atom is also shown in Table I for room temperature. This correction will further increase the difference between theoretical and experimental values.

The open circles in Fig. 1 show the $\langle\alpha\rangle$ values for different isomers with energies close to the ground state. This gives us an idea of the range of the variation of $\alpha$ values one may expect if the structure of the cluster changes. From the figure, we see that the $\langle\alpha\rangle$ values (for the same $n$ ) do have a noticeable dependence on the structure, but it is important to note that all the calculated $\langle\alpha\rangle$ values are above the bulk limit irrespective of the atomic arrangements. It seems that all the isomers with reasonable energies have $\langle\alpha\rangle$ values higher the bulk limit in this regime. From this observation, it is unlikely that the agreement between theory and experiment can be improved by considering isomeric variations.

In the literature, the polarizabilities of semiconductor clusters and quantum dots are sometimes discussed in terms of the size of the gap. Larger polarizabilities are usually correlated with smaller gaps. From perturbation expansions, the polarizability involves a summation over all the occupied and unoccupied states, and there is no reason why the gap alone should dominate. In addition, the oscillator strengths are important. It would nevertheless be nice if the polariz-

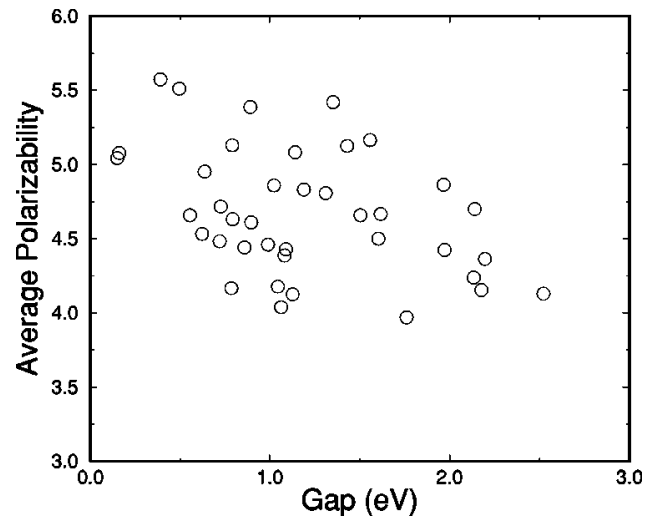

FIG. 2. The calculated average polarizability (in $\AA^{3}$ per atom) plotted against the HOMO-LUMO gap for the $\mathrm{Si}_{n}$ clusters with 9 $\leqslant n \leqslant 28$.

ability can be qualitatively correlated with the gap size. To see if such correlation exists for small $\mathrm{Si}_{n}$ clusters, we plot in Fig. 2 the polarizability as a function of the HOMO-LUMO gap for all the clusters we have calculated. While it is true that clusters with very small HOMO-LUMO gaps (such as those with gaps less than $0.5 \mathrm{eV}$ ) have larger polarizability than those with large gaps (say with gaps bigger $2.5 \mathrm{eV}$ ), the overall distribution is so scattered that the correlation is too weak to be useful in this small $n$ regime. We next see whether $\langle\alpha\rangle$ can be correlated with a simple parameter that characterizes the geometry. To this end, we define a normalized moment $I / I_{o}$, where $I=m \Sigma_{i} r_{i}^{2}$ is the second moment relative to the cluster center of mass, and $I_{o}=\frac{2}{5} \mathrm{Nma}^{2}$ is the moment of inertia of a sphere having the volume of $N$ bulk $\mathrm{Si}$ atoms each of mass $m$. We found that the larger clusters tend to have higher $I / I_{o}$ because they are structurally more elongated. The calculated $\langle\alpha\rangle$ versus $I / I_{o}$ is shown in Fig. 3 for the ground state configurations and we see that clusters with higher $I / I_{o}$ have larger $\langle\alpha\rangle$. This correlation is consistent with the classical picture that more elongated objects are more polarizable.

In summary, we have calculated the dipole moments and the polarizabilities for Si clusters with $n$ from 9 to 28 . The geometry of the clusters have been determined previously by

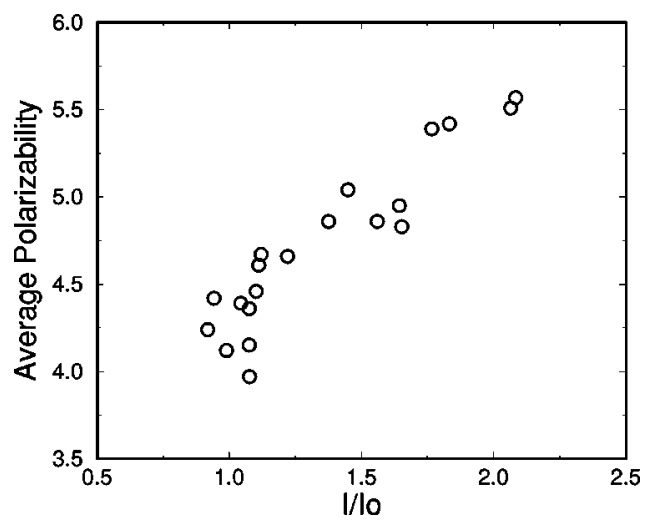

FIG. 3. The calculated average polarizability (in $\AA^{3}$ per atom) plotted against the normalized inertia (see text) for the ground state $\mathrm{Si}_{n}$ clusters with $9 \leqslant n \leqslant 28$. 
advanced optimization techniques. There is a noticable deviation between theoretical and experimental determined polarizabilities and the difference is bigger than the typical error bars quoted in the measurement. The difference is also bigger than what we can usually attribute to the errors introduced in the local density approximations (if the geometry is known; see, for example, the test for $\mathrm{CO}$ ). In other words, the theoretically determined geometry and the measured polarizability have yet to be reconciled in this regime. Recently, Jackson and co-workers [16] have calculated the polarizabilities of Si clusters with $n$ from 10 to 20, and the our results are consistent with theirs. In particular, all calculated $\langle\alpha\rangle$ are higher than the bulk limit. When we consider up to $n=28$, we observe the trend that $\langle\alpha\rangle$ attains a minimum near $n=10$ to 15 and the $\langle\alpha\rangle$ values actually increase again for larger clusters.

We are very grateful to Dr. BiCai Pan and Dr. A. Sieck for providing us with the atomic coordinates of the Si clusters, and many helpful correspondences. C.T.C. thanks Professor K. M. Ho for alerting him to Ref. [16]. This work was partially supported by the Hong Kong Research Grant Council through Grant No. HKUST6128/98P, by the National "Climb' Project of China, by the National Natural Science Foundation of China, and by the Foundation of the Chinese Academy of Science.
[1] J.C. Grossman and L. Mitas, Phys. Rev. Lett. 74, 1323 (1995).

[2] J.C. Grossman and L. Mitas, Phys. Rev. B 52, 16735 (1995).

[3] K. Raghavachari and C.M. Rohlfing, J. Chem. Phys. 89, 2219 (1988)

[4] J.R. Chelikowsky, J.C. Phillips, M. Kamal, and M. Strauss, Phys. Rev. Lett. 62, 292 (1989).

[5] J.R. Chelikowsky and J.C. Phillips, Phys. Rev. Lett. 63, 1653 (1989).

[6] R. Schäfer, S. Schlecht, J. Woenckhaus, and J.A. Becker, Phys. Rev. Lett. 76, 471 (1996).

[7] I. Vasiliev, S. Ögüt, and J.R. Chelikowsky, Phys. Rev. Lett. 78, 4805 (1997).

[8] A. Sieck, D. Porezag, T. Frauenheim, M.R. Pederson, and K. Jackson, Phys. Rev. A 56, 4890 (1997).

[9] K. Jackson, M.R. Pederson, D. Porezag, Z. Hajnal, and T.
Frauenheim, Phys. Rev. B 55, 2549 (1997).

[10] K.M. Ho, A.A. Shvartsburg, B.C. Pan, Z.Y. Lu, C.Z. Wang, J.G. Wacker, J.L. Fye, and M.F. Jarrold, Nature (London) 392, 582 (1998)

[11] A.A. Shvartsburg, M.F. Jarrold, B. Liu, Z.Y. Lu, C.Z. Wang, and K.M. Ho, Phys. Rev. Lett. 81, 4616 (1998).

[12] B. Liu, Z.Y. Lu, B.C. Pan, C.Z. Wang, and K.M. Ho, J. Chem. Phys. 109, 9401 (1998).

[13] A registered software product of Molecular Simulations Inc.

[14] B.C. Pan (private communication).

[15] J.P. Perdew, J.A. Chevary, S.H. Vosko, K.A. Jackson, M.R. Pederson, D.J. Singh, and C. Fiolhais, Phys. Rev. B 46, 6671 (1992).

[16] K. Jackson, M. Pederson, C.Z. Wang, and K.M. Ho, Phys. Rev. A 59, 3685 (1999). 